\title{
Combining CBP Pharmacophore Construction and Molecular Docking to Search for Potential Competitive Inhibitors of Chitin Deacetylase
}

\section{Zizhong Tang ( 14126@sicau.edu.cn )}

Sichuan Agricultural University https://orcid.org/0000-0002-4197-9265

Xiaoli Fu

Sichuan Agricultural University https://orcid.org/0000-0001-7616-5352

Lu Huang

Sichuan Agricultural University

Haoxiang Wang

Sichuan Agricultural University

Biao Tang

Sichuan Agricultural University

Yirong Xiao

Sichuan Agricultural University Hospital

\section{Yujun Wan}

Sichuan Food Fermentation Industry Research and Design Institute

Hui Chen

Sichuan Agricultural University

Huipeng Yao

Sichuan Agricultural University

\section{Zhi Shan}

Sichuan Agricultural University

\section{Gang Wang}

Sichuan Dood Fermentation Industry Research and Design Institute

\section{Research}

Keywords: chitin deacetylase, colletotrichum, virtual screening, docking

Posted Date: November 2nd, 2020

DOI: https://doi.org/10.21203/rs.3.rs-99552/v1 
License: (c) (i) This work is licensed under a Creative Commons Attribution 4.0 International License. Read Full License 


\section{Combining CBP Pharmacophore Construction and Molecular}

\section{Docking to Search for Potential Competitive Inhibitors of Chitin}

\section{Deacetylase}

\section{Zizhong Tang ${ }^{1 * \dagger}$, Xiaoli Fu ${ }^{1 \dagger}$, Lu Huang1, Haoxiang Wang ${ }^{1}$, Biao Tang ${ }^{1}$, Yirong Xiao ${ }^{2}$, Yujun}

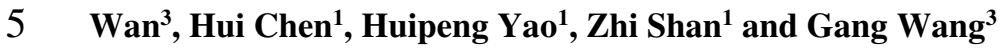

61 College of Life Sciences, Sichuan Agricultural University, Ya'an, 625014, China

72 Sichuan Agricultural University Hospital, Ya'an 625014, China

83 Sichuan Food Fermentation Industry Research and Design Institute, Chengdu 611130, China

$94+$ These authors contributed equally to this work and should be considered co-first authors

$10 *$ Corresponding Author:

11 Zizhong Tang, Tel: +86 0835 2886126; Fax: +86 0835 2886136, Xinkang road, Ya'an, Sichuan,

12625014 , China

13 Email address: 14126@sicau.edu.cn

14 Abstract: Chitin deacetylase (CDA) is a key enzyme for plant pathogens to evade host defense 15 recognition. However, in the study of CDA inhibitors, only chitin deacetylase from colletotrichum 16 lindemuthianum (ClCDA) was found to participate in the reverse hydrolysis reaction in sodium acetate 17 to acetylate free amino sugar residues into $\mathrm{N}$-acetylated forms. Based on this, we selected 10,632 small 18 molecules from the DrugBank database for computer virtual screening to find new potential CDA 19 inhibitors. First, we use the CBP model with ROC $=0.800$ to coarsely screen small molecules. Then 20 we use the LibDock and CDOCKER programs in Discovery Studio 2016 (DS 2016) to dock the best21 matched small molecules to identify interactions with key residues on the active site of ClCDA. Finally, we found two potential compounds with good adaptability, high docking score and important

23 interactions with protein active sites. And we confirm that their structures are stable and have multiple 24 non-bonding interactions with important amino acid sites such as ASP50, TYR145, HIS206 and 25 ZN1255 by MD simulations. Therefore, we conclude that the selected compounds are likely to be new 26 inhibitors of CDA. In this research could provide a valuable resource and guidance for CDA-related 27 inhibitors development. 
28 Keywords: chitin deacetylase; colletotrichum; virtual screening; docking.

\section{Introduction}

In the long struggle between plants and pathogens, plants have evolved a highly efficient and complex immune system, and pattern recognition receptors located on plant cell membrane epitopes play an important role in sensing the presence of pathogens and activating immunity. Chitin is one of the important components of fungal cell wall, and chitin released by pathogenic fungi in the process of infecting host will be recognized by host membrane receptors to induce immune response[1-3]. However there is cumulative evidence that fungi evade plant defense mechanisms by partially deacetylating either their exposed cell wall chitin[4-6]. In this cases, the resulting partially deacetylated oligomers are not well recognized by the specific plant receptors reducing or preventing the elicitation of the defense responses[7]. Currently, antifungals targeting cell walls include $\beta$-D-glucan synthase inhibitor, chitin synthase inhibitors and glycosyl-phosphatidyl Inositol (GPI) anchor pathway inhibitor[8, 9]. Thus, CDA represents a promising target for antifungals.

Chitin deacetylase is one of the members of Carbohydrate esterase 4 superfamily, which can hydrolyze acetyl groups of $\mathrm{N}$-acetylglucosamine units of chitin and chitin oligosaccharides, thus producing acetic acid and chitosan, the poor substrates of chitinase[10]. As important enzyme catalyzing the conversion to chitin to chitosan, chitin deacetylase plays a very important role in agriculture and drug discovery. We can seek chitin deacetylase inhibitors to block the deacetylation modification of chitin by chitin deacetylase, tear apart the cunning camouflage of pathogenic fungi, expose their true state, and make the organisms play a therapeutic role in their own prevention and treatment.

Colletotrichum is a genus of soil-borne plant fungi widely distributed in tropical, subtropical and temperate regions, which often infects crops and induces serious economic losses[11]. In a vote organized by Molecular Plant Pathology magazine in 2012, the pathogenic fungi of the genus Anthrax were promoted as the eighth most important phytopathogenic fungi in the world according to their scientific significance and economic importance[12]. Among the numerous studies on chitin deacetylase, some researchers have found that in $3.0 \mathrm{~mol} / \mathrm{L}$ sodium acetate, $\mathrm{ClCDA}$ can participate in the reverse hydrolysis reaction, acetylate the free amino sugar residues into $\mathrm{N}$-acetylated form, and some studies have confirmed that acetate plays a competitive inhibitory role in this process. This makes it possible to control plant pathogens by inhibiting chitin deacetylase[13-15]. 

resolution technology (X-ray diffraction, nuclear magnetic resonance). The use of computational

59 techniques in drug discovery and development has become the most effective method. Ligand-based 60 virtual screening of drugs can efficiently screen potential compounds from a large number of compounds 61 through the interaction between proteins and small molecule compounds, avoiding blind screening, 62 thereby reducing human, financial and time costs[16]. This study will use DS2016 software to further 63 explore competitive inhibitors of chitin deacetylase on the basis of docking acetate and other molecules 64 with chitin deacetylase. The virtual screening flow chart is shown in Fig. 1.

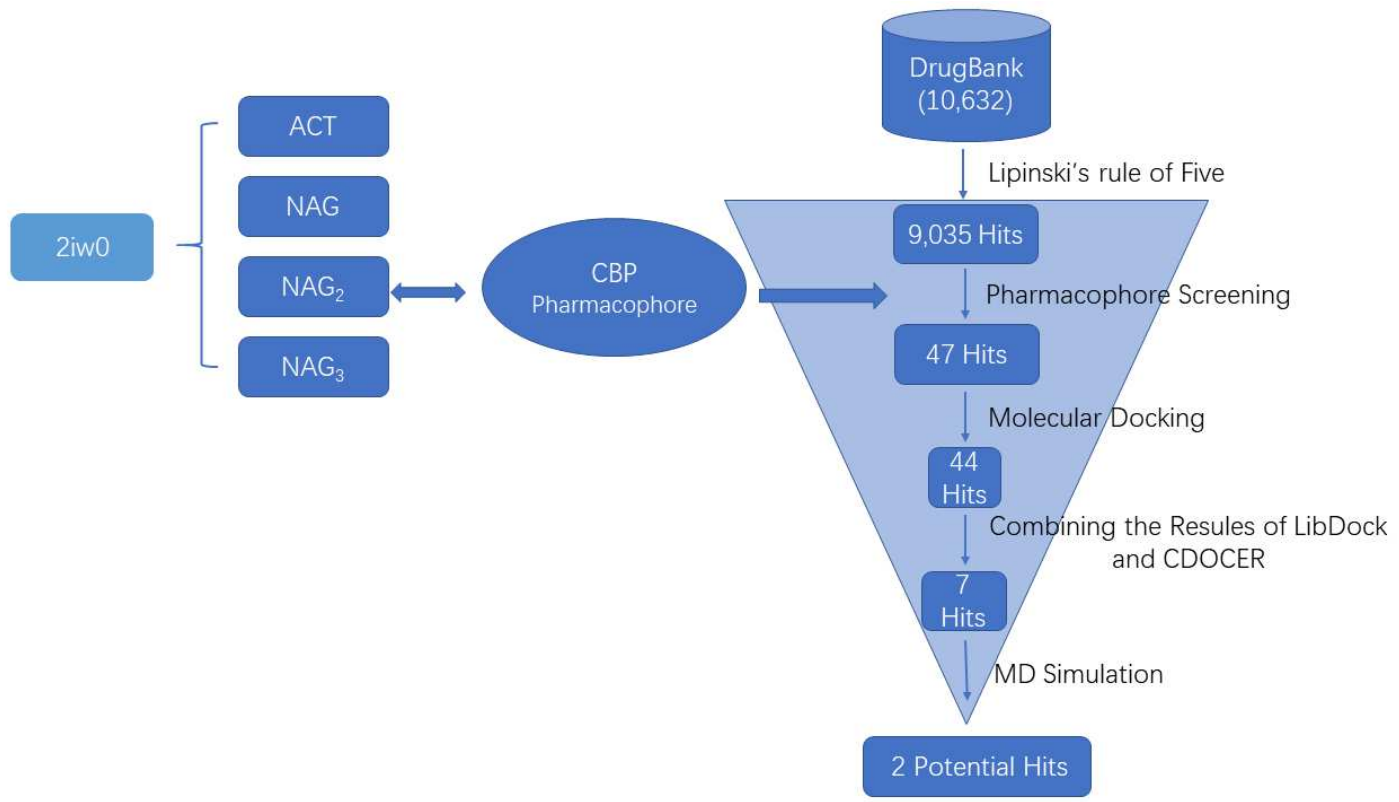

66 Fig. 1. Schematic representation of the virtual screening process implemented in the identification of 67 ClCDA inhibitors. 2iw0: crystal structure of ClCDA; ACT: acetate ion; NAG: chitin monomer form; 68 NAG2: chitin dimer form; NAG3: trimeric form of chitin.

\section{Materials and Methods}

\section{Data Collection and Preparation}

The X-ray crystal structure of ClCDA (PDB ID: 2iw0) was downloaded from the RCSB Protein

72 Data Bank (www.rcsb.org). According to the relevant literature, it is known that the ClCDA catalytic 73 subunit is generated from zinc-binding triplets, which are composed of two histidine (His104, His108)

74 and aspartate (Asp50)[17]. Therefore, after removing the original ligand from the complex, ASP50, 75 HiS104, HiS108 and Zn1255 were set as active sites and the active radius was set to $10 \AA$. Then 2 iw0 

form of chitin $\left(\mathrm{NAG}_{3}\right)$ respectively for LibDock molecular docking. Finally, the docking results of 2iw0-

78 ACT was used as receptor, and three forms of $\mathrm{N}$-acetylglucosamine chitosan were used as ligands for LibDock molecular docking respectively.

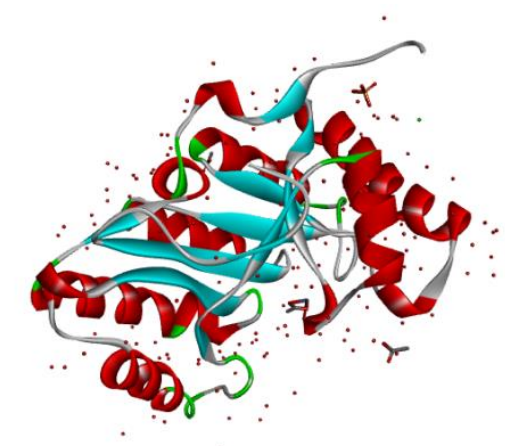

Fig. 2. Crystal structure of chitin deacetylase (2iw0).

\section{Pharmacophore Model Generation}

The highest scoring $\mathrm{NAG}_{2}-2 \mathrm{iw} 0$ conformation was selected according to the results of LibDock docking to construct a receptor-ligand complex based pharmacophore model (CBP).The pharmacophore model generates crystal complexes utilizing known ClCDA (PDB ID: 2iw0) and ligand ( $\mathrm{NAG}_{2}$ ), and chooses both as receptors and ligands, respectively, to build the CBP model within the protocol of 'receptor-ligand pharmacophore model generation' in DS . Specifically, the maximum hydrophobic distance was set to5.5 $\AA$ and the maximum hydrogen bond distance was set to $3.0 \AA$. Other parameters such as 'mode' and 'docking' were set as 'fast' and 'rigid', respectively[18]. In order to verify the selectivity of the obtained pharmacophore model, $\mathrm{ACT}, \mathrm{NAG}$ and $\mathrm{NAG}_{3}$ were used as active ligands of CBP for model validation, and 38 compounds in the ClCDA-bait set were randomly selected as inactive ligands. The optimal model was selected and virtual screening was performed using the Drugbank database to identify new potential chitin deacetylase inhibitors [16, 19]. 


\section{H-Bonds Donor \\ Acceptor}

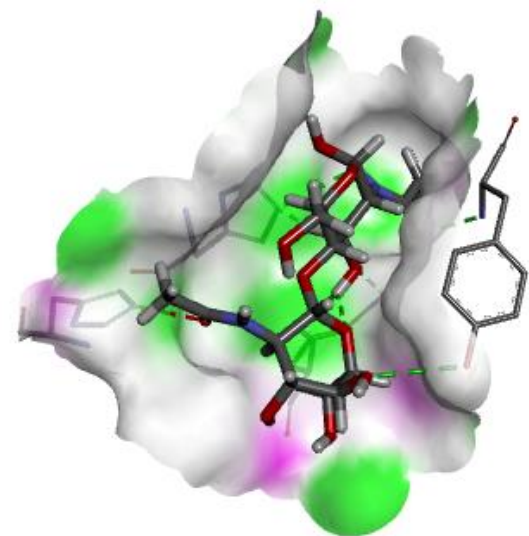

Fig. 3. Hydrogen bond diagram of $2 \mathrm{iw} 0-\mathrm{NAG}_{2}$ interaction.

\section{Molecular Docking}

Because the number of small molecules in DrugBank database is too large, after screening with pharmacophore, there are 7794 remaining small molecules, we use the LibDock molecular docking method to conduct the next round of screening. In this round of screening, the receptor is ClCDA (2iw0), the ligand is Best fit view small molecule obtained after the last round of pharmacophore screening, ASP50, HiS104, HiS108, Zn1255 are set as active sites, and the active radius is set as $10 \AA$. Virtual screening was carried out by docking all the prepared ligands at the defined active site using Libdock. Based on the Libdock score, all the docked poses were ranked and grouped by name. All compounds were ranked according to their Libdock score.

LibDock is a fast rigid docking method using the hot zone map of the active sites of receptor molecules, while CDOCKER is an implementation of a CHARMm based docking tool. The receptor is held rigid while the ligands are allowed to flex during the docking process. For each complex pose, the CHARMm energy (interaction energy plus ligand strain) and the interaction energy, which indicate ligand binding affinity, are calculated[20]. The combination of LibDock and CDOCKEER can make up for the shortcomings of both sides and screen target small molecules quickly and efficiently. Therefore, we used the screening method of CDOCKER molecular docking to screen the small molecules screened by LibDock for another round of screening.

CDOCKER module of Discovery Studio was used for molecular docking study. The CHARMm forcefield was used for receptors and ligands. The binding site spheres of ligands and receptors were defined as the regions that come within radius $10 \AA$ from the geometric centroid of the ligands ASP50, 
114 HiS104, HiS108 and Zn1255, respectively. During the docking process, the ligands were allowed to bind

115 to the residues within the binding site spheres[20].

116

117

118

119

120

121

123

124

125

126

\section{Results}

128

129

130

131

132

133

134

135

136

137

\section{Molecular Dynamics Simulations}

Molecular dynamics (MD) simulations were performed using DS 2016 Standard Dynamics Cascade and Dynamics package. Samples of ligand-receptor complexes were applied with the CHARMM polar hydrogen force filed and solvated by applying explicit periodic boundary in a solvation model before running MD simulations. MD simulations were conducted under the setting parameters, which were listed as follows: steepest descent of energy minimization was 500, steps of conjugate gradient minimization were 500 , the system was heated from $50 \mathrm{~K}$ to $300 \mathrm{~K}$ within 2 ps, and steps of equilibration were 1000 . The simulations were performed with a total production time of $200 \mathrm{ps}$. For other parameters, we adopted default setting values. We used the functions of Analyze Trajectory to analyze root mean square deviations (RMSDs) of protein-ligand complexes and ligands, total energies and potential energies of protein-ligand complex, after MD simulation[21].

Identification of Substrate-Binding Region

ClCDA is a member of the Carbohydrate esterase 4 superfamily, whose members share a conserved region in their primary structure that is recognized as a catalytic subunit[22]. It is known that the ClCDA catalytic subunit is generated from a zinc-binding triad consisting of two histidines (His 104, His108) and aspartate (Asp50)[22]. Therefore, we used the zinc-binding triplet as the active center for LibDock docking with the substrates of ClCDA (NAG, $\mathrm{NAG}_{2}, \mathrm{NAG}_{3}$ ) and competitive inhibitors (ACT). At the same time, in order to intuitively demonstrate the mechanism of action of the inhibitors, we docked the substrates again while retaining the docking of the inhibitors. The docking results are shown in Table 1 and 2 below.

Table 1. Molecular docking results based on active groups generated by catalytic subunits.

\begin{tabular}{|c|c|c|c|c|}
\hline \multirow{3}{*}{ Name } & \multirow{3}{*}{ ligand non-bond monitor } & \multirow{3}{*}{ Interaction } & Absolute & LibDock \\
\hline & & & & \\
\hline & & & Energy & Score \\
\hline
\end{tabular}




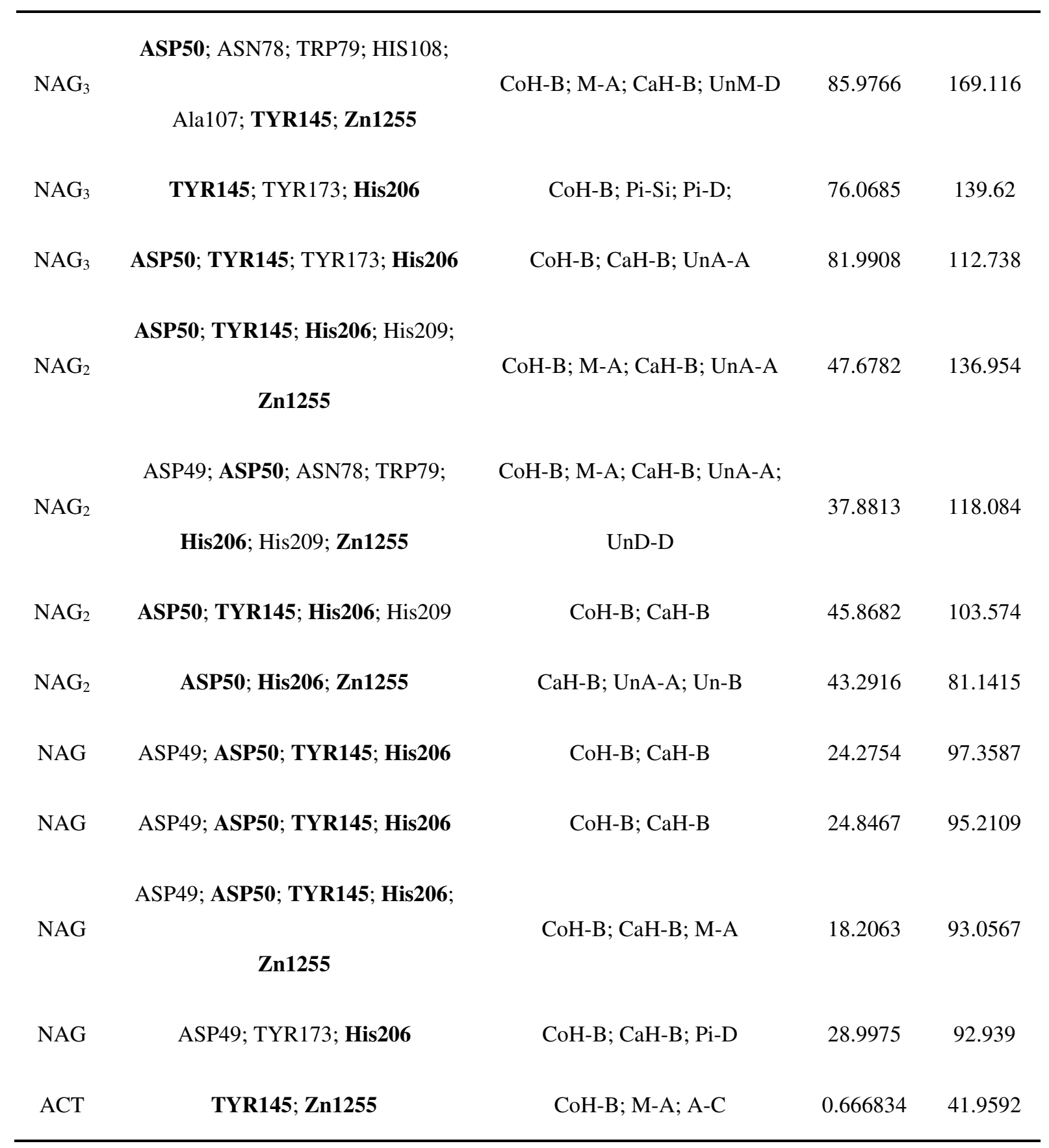

138 CoH-B: Conventional Hydrogen Bond; M-A: Metal-Acceptor; CaH-B: Carbon Hydrogen Bond; UnM-

139 D: Unfavorable Metal-Donor; Pi-Si: Pi-Sigma; Pi-D: Pi-Donor Hydrogen Bond; UnA-A: Unfavorable

140 Acceptor-Acceptor; UnD-D: Unfavorable Donor-Donor; Un-B: Unfavorable Bump; A-C:Attractive 141 Charge.

142 Table 2. Molecular docking results based on the active group generated by adding ACT1256 catalytic subunit. 


\begin{tabular}{|c|c|c|c|c|}
\hline \multirow{3}{*}{ Name } & \multirow{3}{*}{ ligand non-bond monitor } & \multirow{3}{*}{ Interaction } & \multirow{3}{*}{$\begin{array}{l}\text { Absolute } \\
\text { Energy }\end{array}$} & \multirow{3}{*}{$\begin{array}{l}\text { LibDock } \\
\text { Score }\end{array}$} \\
\hline & & & & \\
\hline & & & & \\
\hline \multirow{3}{*}{$\mathrm{NAG}_{3}$} & ASP50; ASN78; TRP79; Ala107; & vdW; CoH-B; CaH-B; UnD-D; & & \\
\hline & & & 67.0774 & 137.545 \\
\hline & HIS108; TYR145; His206 & Un-B & & \\
\hline \multirow{3}{*}{$\mathrm{NAG}_{3}$} & ASP50; TRP79; Ala107; TYR145; & & & \\
\hline & & CoH-B; CaH-B; UnD-D & 73.7395 & 132.644 \\
\hline & TYR173; His206; ACT1256 & & & \\
\hline $\mathrm{NAG}_{3}$ & ASP50; TYR145; His206 & CoH-B; CaH-B; Pi-D & 72.5358 & 125.602 \\
\hline \multirow[t]{2}{*}{$\mathrm{NAG}_{2}$} & ASP50; TRP79; HIS108; TYR173; & CoH-B; CaH-B; Pi-D & 29.6294 & 91.8633 \\
\hline & ACT1256 & & & \\
\hline \multirow{2}{*}{$\mathrm{NAG}_{2}$} & ASP50; ASN78; TRP79; TYR145; & CoH-B; P-LP; CaH-B; UnA-A; & 364111 & 01252 \\
\hline & TYR173; His206; His209 & UnD-D & & \\
\hline \multirow[t]{2}{*}{$\mathrm{NAG}_{2}$} & ASP50; ASN78; TYR145; His206; & CoH-B; CaH-B & 34.9803 & 90.6119 \\
\hline & His 209 & & & \\
\hline $\mathrm{NAG}_{2}$ & $\begin{array}{c}\text { ASP50; ASN78; TRP79; TYR145; } \\
\text { His206; His209 }\end{array}$ & $\mathrm{CoH}-\mathrm{B} ; \mathrm{CaH}-\mathrm{B}$ & 39.0936 & 90.0171 \\
\hline \multirow[t]{2}{*}{ NAG } & ASP50; ASN78; TRP79; HIS108; & CoH-B; CaH-B; UnD-D & 14.1647 & 89.6868 \\
\hline & His206 & & & \\
\hline NAG & ASP50; ASN78; His206; His209 & $\mathrm{CoH}-\mathrm{B} ; \mathrm{CaH}-\mathrm{B}$ & 27.6388 & 85.2853 \\
\hline NAG & ASP50; His206; His209 & CoH-B; CaH-B; UnA-A & 24.4608 & 83.7609 \\
\hline NAG & ASP50; ASN78; TRP79; His209 & $\mathrm{CoH}-\mathrm{B} ; \mathrm{CaH}-\mathrm{B}$ & 18.1118 & 82.7795 \\
\hline
\end{tabular}

144 CoH-B: Conventional Hydrogen Bond; M-A: Metal-Acceptor; CaH-B: Carbon Hydrogen Bond; UnM-

145 D: Unfavorable Metal-Donor; Pi-Si: Pi-Sigma; Pi-D: Pi-Donor Hydrogen Bond; UnA-A: Unfavorable 
From the docking results, it is noteworthy that ASP50, TYR145, His206, Zn1255 appear at high frequency in the docking of $\mathrm{NAG}, \mathrm{NAG}_{2}, \mathrm{NAG}_{3}$ (Table 1). These groups are likely to be important sites for the binding of ClCDA and chito-oligosaccharides, which means that if these sites are bound by other substances, they will competitively inhibit chito-oligosaccharides and then inhibit ClCDA. This is of great breakthrough significance in inhibiting the deacetylation modification of chitin in the cell wall of pathogenic fungi, enabling the chitinase secreted by host cells to successfully recognize and hydrolyze chitin, thus controlling the infection of plant pathogenic fungi, and finding new inhibitors.

By comparing the docking results of whether the catalytic subunit contains ACT or not, it was found that the docking results of $\mathrm{NAG}, \mathrm{NAG}_{2}$ and $\mathrm{NAG}_{3}$ after adding $\mathrm{ACT}$ were not as good as before. Absoiute Energy and LibDock Score scores were lower than previous values. LibDock score is a comprehensive representation of van der Waals forces, hydrogen bonds, PI interactions, and other parameters. Higher the LibDock score and absolute energy means a high chance of ligand-protein binding[23, 24]. Analysis of the interaction groups revealed that none of the conformations had an effect on Zn1255 after the addition of ACT. According to the data, chitin deacetylases are representative members of the CE-4 family, which usually rely on metal-dependent mechanisms for acid/base catalysis[17]. It is inferred that Zn1255 plays an important role in deacetylation of chitin deacetylase-bound substrates, and the addition of ACT may block the interaction between $\mathrm{Zn}^{2+}$ and substrates, perhaps, $\mathrm{Zn}^{2+}$ chelators could act as inhibitors of $\mathrm{CDA}[25]$. This provides important site information for searching for new inhibitors.

\section{Generating Receptor-ligand Pharmacophores}

169 docking is often discounted because these programs can place compounds anywhere in the binding site,

170 and the corresponding scoring equation often cannot find the most likely binding site. But in most cases,

171 for a given binding site, which interaction plays a key role in ligand-receptor interaction is often 172 known[26]. Because, from the complex structure, we can get the groups and their spatial distribution 173 which contribute greatly to the activity of the inhibitors. In this case, the experience-based discovery of 174 binding sites and known binding modes can be considered in the docking process to create a 
175 pharmacophore model for docking. This will lead potential inhibitors to bind to known, energetically

176 favorable interactions.

177 As far as the LibDock docking results of the catalytic subunit are concerned (Table 1), the first 178 conformation of $\mathrm{NAG}_{2}$ is linked to all the groups recurring at present, and the higher scores of Absolute

179 Energy and LibDock Score are only second to highest scores. This means that the concept is well 180 integrated with ClCDA. Therefore, it is excellent to construct a pharmacophore model (CBP) based on 181 receptor-ligand complexes using this conformation.

182 A total of 10 pharmacophore models were generated by CBP operation based on $\mathrm{NAG}_{2}$ 183 conformation. Fifty-six features were found in the ligands: HB_ACCEPTOR: 31; HB_DONOR: 25, and 184 eight features of matching receptor-ligand interaction: AAAAADDD. The top 10 models with the highest 185 Selectivity Score were retained after combining their permutations, as shown in Table 3. In combination 186 with several eigenvalues of Sensitivity, Specificity, ROC, Selectivity Score and Feature Set, 187 Pharmacophore_01 pharmacophore model is the best, with equal number of Character Set hydrogen 188 acceptor donors and the best sensitivity and specificity. Although the ROC value is only 0.800 and less 189 than 0.822 , it is also a good score, and its Selectivity Score is far superior to other pharmacophores. 190 Therefore, Pharmacophore_01 pharmacophore was selected as a model for the next potential inhibitor 191 screening.

Table 3. ROC and Selectivity Score values of 10 pharmacophore model characteristics.

\begin{tabular}{ccccccc}
\hline & Number of & & & & Selectivity \\
Pharmacophore & & Feature Set & Sensitivity & Specificity & ROC & \\
& Features & & & & Score \\
\hline Pharmacophore_01 & 6 & $\mathrm{~A}_{1} \mathrm{~A}_{4} \mathrm{~A}_{5} \mathrm{D}_{6} \mathrm{D}_{7} \mathrm{D}_{8}$ & 0.66667 & 0.93333 & 0.800 & 12.274 \\
Pharmacophore_02 & 6 & $\mathrm{~A}_{1} \mathrm{~A}_{3} \mathrm{~A}_{4} \mathrm{~A}_{5} \mathrm{D}_{6} \mathrm{D}_{7}$ & 0.66667 & 0.93333 & 0.822 & 11.361 \\
Pharmacophore_03 & 6 & $\mathrm{~A}_{1} \mathrm{~A}_{2} \mathrm{~A}_{4} \mathrm{~A}_{5} \mathrm{D}_{6} \mathrm{D}_{8}$ & 0.66667 & 0.93333 & 0.822 & 11.361 \\
Pharmacophore_04 & 5 & $\mathrm{~A}_{1} \mathrm{~A}_{4} \mathrm{D}_{6} \mathrm{D}_{7} \mathrm{D}_{8}$ & 0.66667 & 0.73333 & 0.744 & 10.760 \\
Pharmacophore_05 & 5 & $\mathrm{~A}_{1} \mathrm{~A}_{5} \mathrm{D}_{6} \mathrm{D}_{7} \mathrm{D}_{8}$ & 0.66667 & 0.73333 & 0.656 & 10.760 \\
Pharmacophore_06 & 5 & $\mathrm{~A}_{4} \mathrm{~A}_{5} \mathrm{D}_{6} \mathrm{D}_{7} \mathrm{D}_{8}$ & 0.66667 & 0.73333 & 0.722 & 10.760 \\
\hline
\end{tabular}




\begin{tabular}{lcccccc}
\hline Pharmacophore_07 & 6 & $\mathrm{~A}_{1} \mathrm{~A}_{2} \mathrm{~A}_{3} \mathrm{~A}_{4} \mathrm{~A}_{5} \mathrm{D}_{6}$ & 0.66667 & 0.93333 & 0.822 & 10.447 \\
Pharmacophore_08 & 5 & $\mathrm{~A}_{1} \mathrm{~A}_{3} \mathrm{~A}_{5} \mathrm{D}_{6} \mathrm{D}_{7}$ & 0.66667 & 0.66667 & 0.756 & 9.8460 \\
Pharmacophore_09 & 5 & $\mathrm{~A}_{2} \mathrm{~A}_{4} \mathrm{~A}_{5} \mathrm{D}_{6} \mathrm{D}_{8}$ & 0.66667 & 0.86667 & 0.767 & 9.8460 \\
Pharmacophore_10 & 5 & $\mathrm{~A}_{1} \mathrm{~A}_{4} \mathrm{~A}_{5} \mathrm{D}_{6} \mathrm{D}_{7}$ & 0.66667 & 0.73333 & 0.767 & 9.8460 \\
\hline
\end{tabular}

193

$\mathrm{A}=$ Acceptor; $\mathrm{D}=$ Donor.

194

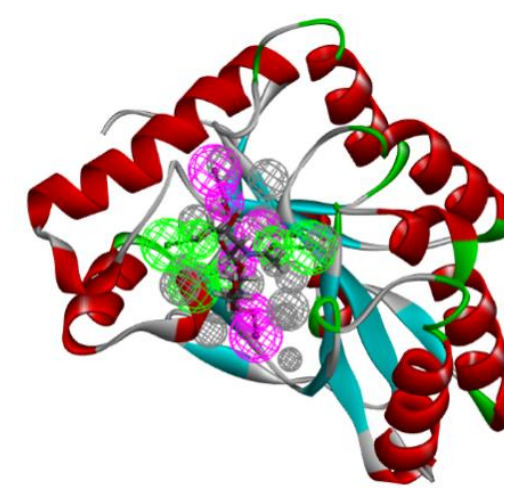

(A)

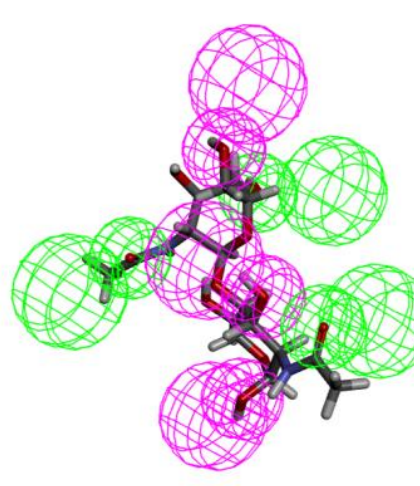

(B)

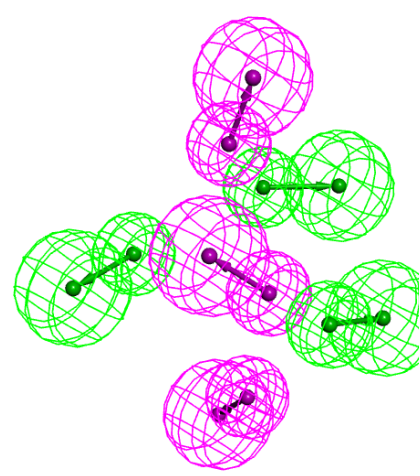

(C)

195 Fig. 4. (A) Chitin deacetylase crystals (PDB code: 2iw0) with interaction diagram of co-crystalline

196 ligand $\mathrm{NAG}_{2}$ and pharmacophore_01, (B) co-crystalline ligand $\mathrm{NAG}_{2}$ and pharmacophore_01, (C)

197 pharmacophore_01.

198

199

200

201

202

203

204

\section{CBP Pharmacophore Model-Based Virtual Screening}

Fit value is an index to measure the overlap between pharmacophore characteristics and molecular chemical characteristics, which is helpful to understand the chemical significance of pharmacophore hypothesis[16, 17]. We achieved 10,632 small molecules from the DrugBank database for virtual screening. Finally, 49 HIT compounds mapped to the pharmacophore model Pharmacophore_01 were retrieved according to Best Fit Value in the docking results. The obtained compounds matched well with the CBP model. 
The key characteristic of a good docking program is its ability to reproduce the experimental 207 binding modes of ligands. To test this, a ligand is taken out of the X-ray structure of its protein-ligand 208 complex and docked back into its binding site. The docked binding mode is then compared with the experimental binding mode, and a root-mean-square distance (RMSD) between the two is calculated; a 210 prediction of a binding mode is considered successful if the RMSD is below a certain value (usually 2.0

$211 \AA$ A [27]. In this study, docking analysis of the active site of ClCDA was performed using DS 2016.

212 Ligands in protein 2iw0 were extracted. The docking method adopts two docking methods, LibDock and 213 CDOCKEER. Subsequently, the binding positions of the docked compounds were compared with the 214 ligands in the crystal complexes, and the RMSD values deviations were calculated to be 0.2587 and 2150.3410 , respectively. It can be seen from Fig. 5 that the ligands docked by these two docking methods 216 are well aligned with the ligands in crystal complexes, which proves the accuracy and reliability of these 217 two docking methods.

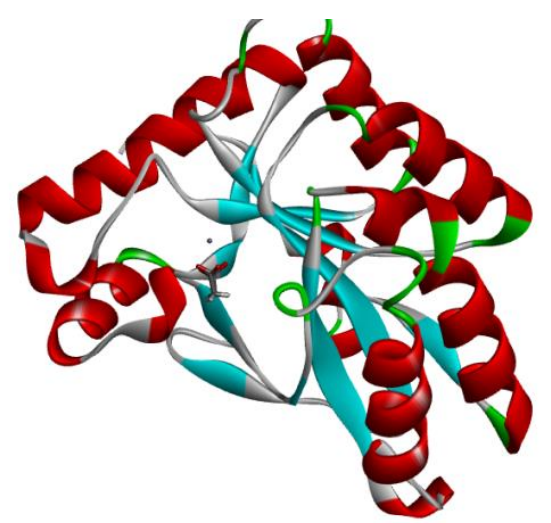

(A)

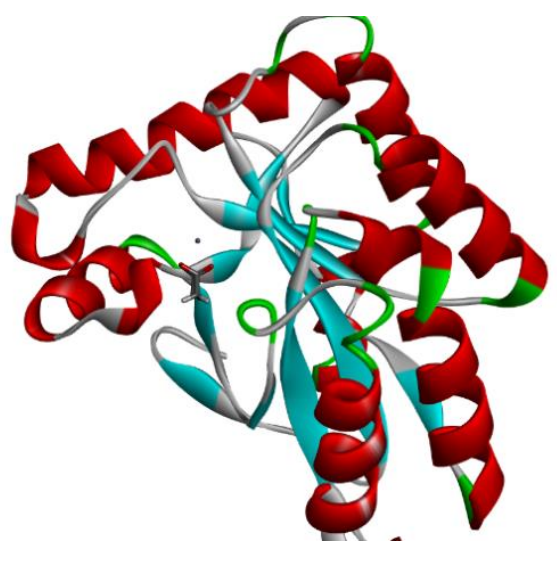

(B)

218 Fig. 5. Alignment of the docked ligands with the ligands in the crystallographic complex. (A) The

219 ligand by the LibDock docking method; (B) The ligand by the CDOCKER docking method.

Receptor-based virtual screening of 49 molecules recovered after pharmacophore-based screening was performed using the LibDock method. Compounds with successful docking were selected, and the selected screened hits were then docked accurately in the CDOCKER module. Finally, the CDOCKER module calculated 44 HIT compounds as targets. We selected the top 20 molecules with the highest scores of LibDock Score and -CDOCKER ENERG, respectively, and found seven common small molecules from them. The docking results of 7 small molecules are as follows: Table 4, Fig. 6. 


\begin{tabular}{|c|c|c|c|c|}
\hline \multirow[b]{2}{*}{ Name } & \multirow[b]{2}{*}{ Structural Formula } & LibDock & -CDOCKER & \multirow[b]{2}{*}{ Fit Valuc } \\
\hline & & Score & ENERG & \\
\hline $\mathrm{ACT}$ & & 41.9592 & 42.7319 & \\
\hline DB02470 & & 111.272 & 39.7564 & 3.62138 \\
\hline DB02824 & & 112.136 & 64.0163 & 2.83566 \\
\hline DB03227 & & 112.370 & 36.5474 & 2.80273 \\
\hline DB03846 & & 124.264 & 37.1136 & 2.88979 \\
\hline
\end{tabular}


<smiles>C=CC1=CC(=C)C([C@H]2C[C@H](C)C(CC(C)(C)C)N2)C=C1C=C</smiles>

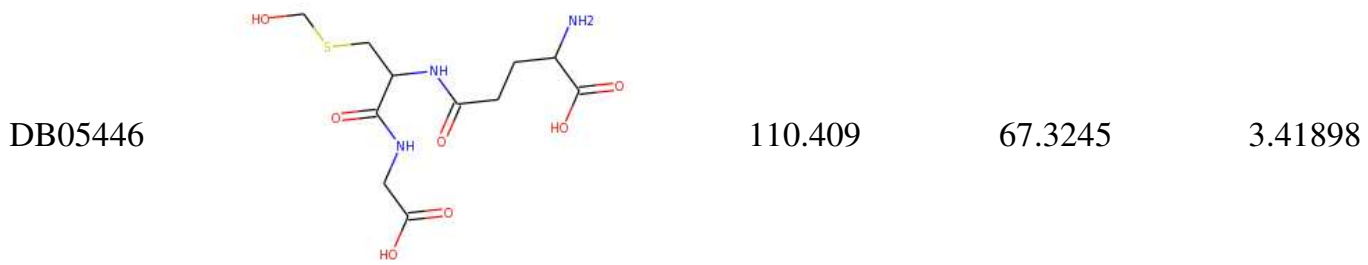

DB11296<smiles>C=C(C)C(CCCCCC)CC(=CC(C)=CC)CC1=CC=CC1</smiles>

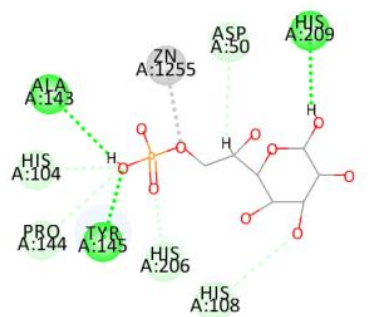

Interactions

Conventional Hydrogen Bond $\square$ Carbon Hydrogen Bond

$\square$ Metal-Acceptor

(A)

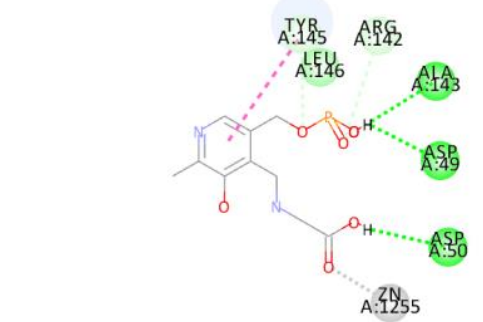

Interactions

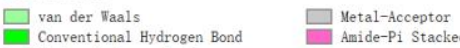

$\square$ Carbon Hydrogen Bond

(B) 


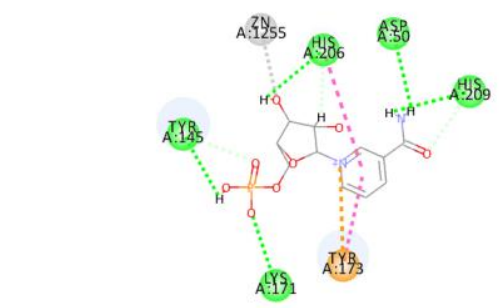

Interactions

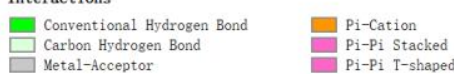

(C)

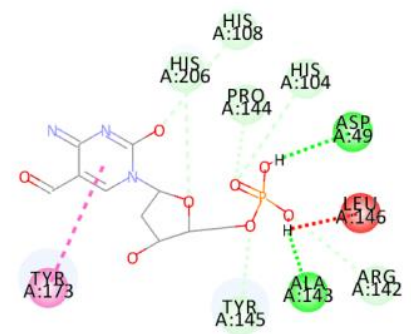

Interactions

Conventional Hydrogen Bor
Carbon Hydrogen Bond

Unfavorable Donor-Donor

(E)

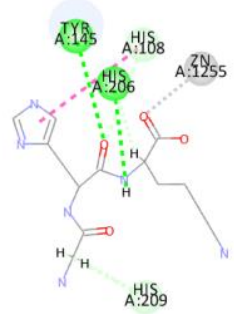

Interactions

Conventional Hydrogen Bo

Carbon Hydrogen Bond

$\square$ Metal-Acceptor
Pi-Pi T-shaped

(G)

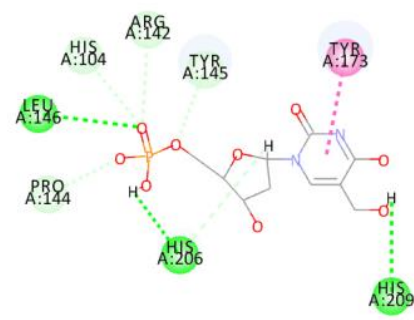

Conventional Hydrogen Bond

(D)

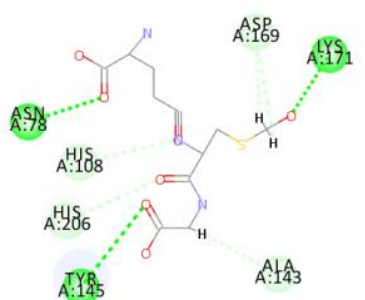

Interactions
Conventional Hydrogen Bond $\quad \square$ Carbon Hydrogen Bond

(F)

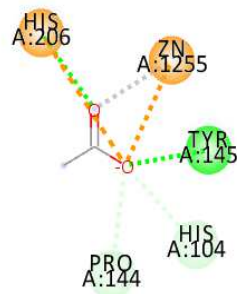

Interactions

Attractive Charge

Conventional Hydrogen Bond

Carbon Hydrogen Bond Metal-Acceptor

(H)

228 Fig. 6. The receptor-ligand interaction of screening compound with the 2 iw0 active site. (A)

229 DB02470 Receptor-ligand interaction with 2iw0 active site. (B) DB02824 Receptor-ligand interaction with 2iw0 active site. (C) DB03227 Receptor-ligand interaction with 2iw0 active site.

231 (D) DB03846 Receptor-ligand interaction with 2iw0 active site. (E) DB04603 Receptor-ligand interaction with 2iw0 active site. (F) DB05446 Receptor-ligand interaction with 2iw0 active site.

233 (G) DB11296 Receptor-ligand interaction with 2iw0 active site. (H) ACT Receptor-ligand interaction with 2 iw0 active site. 
It is not difficult to find through the receptor-ligand interaction of screening compound with the

236 2iw0 active site that only DB02470 and DB03227 interact with the active groups ASP50, TYR145,

237 His206, Zn1255. Compound DB02470 formed generated hydrogen bond with ASP50, TYR145, and

238 HIS206 and generated metallic bond interactions with ZN1255as depicted in Fig. 6(A); Compound

239 DB03227 formed $\pi-\pi$ stacking interactions with TYR173, generated hydrogen bond with ASP50,

240 TYR145, and HIS206 and generated metallic bond interactions with ZN1255 as depicted in Fig. 6(C).

\section{Molecular Dynamics Simulations}

242 Molecular dynamics is the pivotal theoretical approach which can be utilized to gain molecular 243 insight into the stability of the binding pose of the screened molecules in the active site[28]. MD 244 simulations yield energetically favorable conformations by optimizing a protein-ligand complex, which 245 is needed to understand protein-ligand interactions and ligand binding induced structural changes[29].

246 So, according to the results of molecular dynamics simulation, the binding stability of the selected 247 compounds can be verified. The RMSD is commonly used as an indicator of convergence of the structure 248 towards an equilibrium state and is most meaningful for low values. To evaluate the stability of 2iw0 249 ligand complexes under dynamic conditions, we performed molecular dynamics (MD) simulations using 250 DS. Preliminary conformations were obtained by CDOCKER molecular docking experiments. We 251 sampled 100 data points by setting a regular interval from the 200ps simulation trajectory. RMSDs of 252 protein-ligand complexes are shown in Fig. 7, The average value of RMSD for each ligand was calculated 253 over the simulation trajectory. The average RMSD value of protein-ligand complex with DB03227, 254 DB02470 or $2 \mathrm{iw} 0$ was $1.11528 \AA, 0.988519 \AA$ and $0.97396 \AA$, respectively. The RMSD trajectory of the 255 complex was more equilibrium after 100ps, compared with 2iw0. The total energies and potential 256 energies of ligand-protein complexes were approximately identical to each other for the 200ps simulation 257 (shown in Fig. 8 and Fig. 9). Among them, DB03227 has the lowest total energy and potential energies 258 with the receptor. In addition, through molecular dynamics simulation, DB02470 and DB03227 both 259 formed hydrogen bonds with water molecules (shown in Fig. 10). These hydrogen bonds may contribute 260 to the stability of the complexes. Combined with each evaluation index, these two compounds may 261 interact stably with $2 \mathrm{iw} 0$ and have potential negative regulatory effects on $2 \mathrm{iw} 0$. 


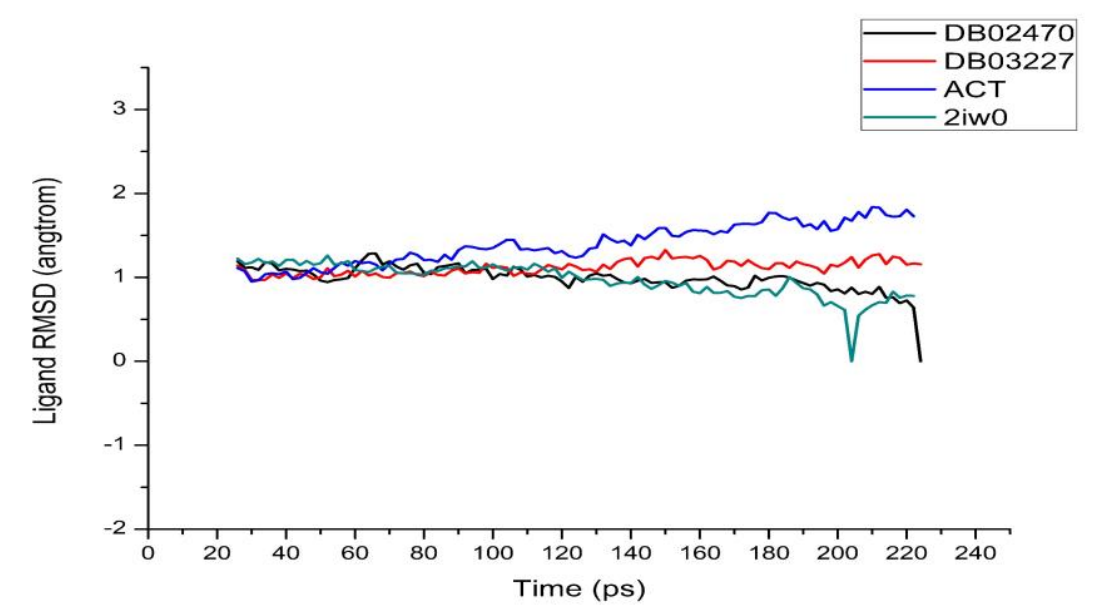

262 Fig. 7. The RMSD values of protein-ligand complexes during MD simulation.

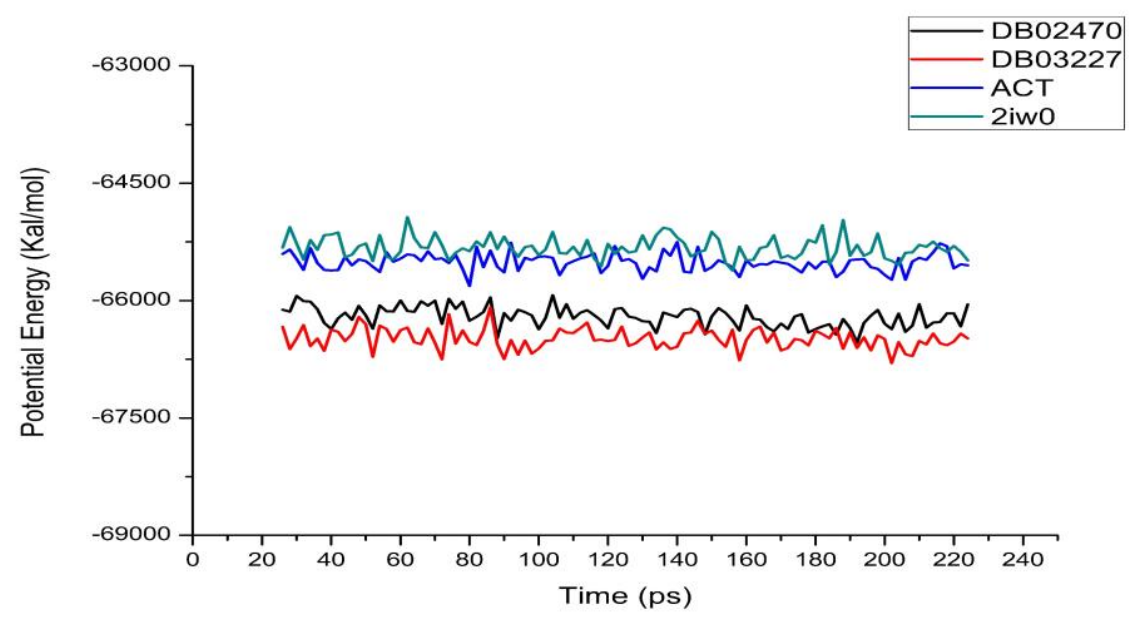

263 Fig. 8. Potential energies of protein-ligand complexes during MD simulation. 


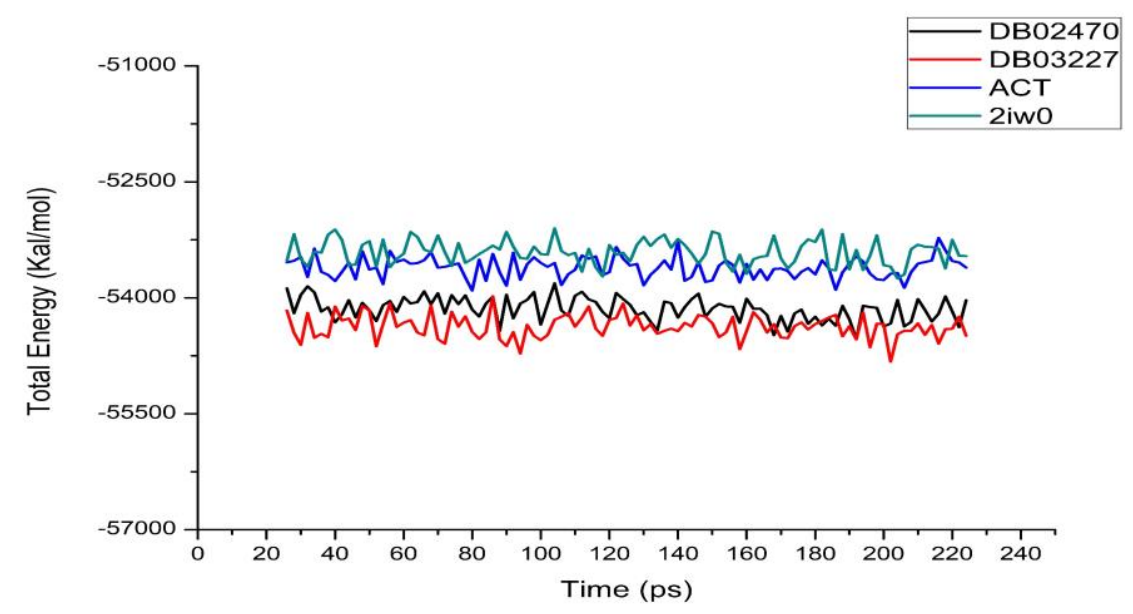

264 Fig. 9. Total energies of protein-ligand complexes during MD simulation.

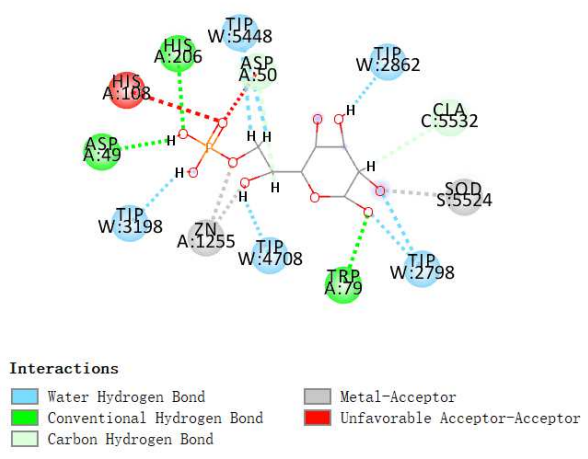

(A)
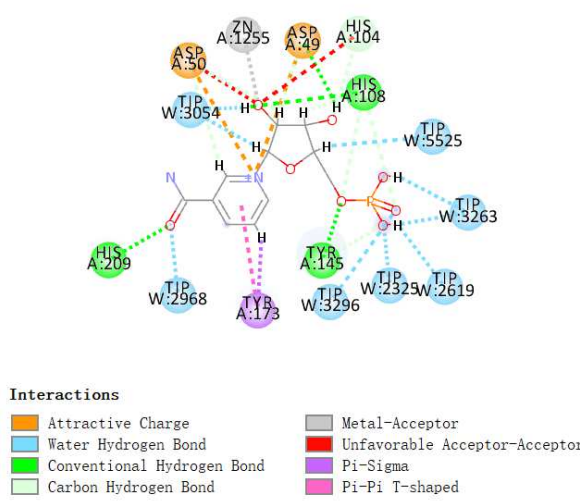

(B)

265 Fig. 10. The compounds were screened for receptor-ligand interaction with the $2 \mathrm{iw} 0$ active site after

266 MD simulation. DB02470 on the left and DB03227 on the right.

\section{Discussion and Conclusions}

In pathogenic plants, $\mathrm{CDA}$ is a heavily glycosylated secreted enzyme allegedly playing a role in the host-pathogen interaction, deacetylating the chitin oligomers resulting from the activity of plant

270 chitinases on the fungal cell walls, thereby evading plant immune defense[7]. Therefore, to find an

271 inhibitor of CDA to weaken its activity is a promising target to resist the infection of phytopathogenic

272 fungi. However, there have been few reports on CDA inhibitors. Only 1 inhibitor, acetate ion (ACT), the

273 ligand selected as the reference in this study, has been part of relatively mature research until now. 
In this study, 10,632 small molecules taken from the DrugBank database for virtual screening, was followed by CBP pharmacophore, LibDock, CDOCKER and molecular dynamics simulation. LibDock and CDOCKER scores unfolded degree of energy optimization and stability of the conormation. High LibDock and CDOCKER score compounds illustrated better energy optimization and a stable conformation than lower score achievers[30]. After calculation by the LibDock and CDOCKER module, 44 compounds showed to be capable to bind stably with ClCDA. Besides, among these ligands, 7 compounds had higher LibDock and CDOCKER scores than ACT (LibDock score: 41.9592; CDOCKER score: -42.7319$)$, indicating that these 7 compounds could form a more stable complex with ClCDA with better energy optimization compared with ACT.

Then, the chemical structures of the 7 compounds were analyzed by molecular structure inspection. The 7 complexes that CICDA combined with 7 candidacies have more chemical bonds than ACT (show in Fig. 6), which again indicates that these 7 compounds could bind with ClCDA at active site more stably. In addition, DB02470 and DB03227 can interact with all the active groups ASP50, TYR145, His206, Zn1255, which again demonstrates the reliability of again demonstrates the reliability of the above suspected active sites, and they may contribute to competitive inhibition of activity of ClCDA.

Finally, their stability in the natural environment were assessed performing molecular dynamics simulation, and it is computational results showed that RMSD, potential energy and total energy of these ligand-CICDA complexes tend to stability with time (show in Fig. 7, 8 and 9), which suggested that these

2922 complexes could exist in the natural environment stably. Molecular dynamics module computation confirmed that RMSD of DB03227 and DB02470 were obviously lower than the reference ligand ACT,

294 which demonstrates these 2 compounds may have a higher stability with CICDA compared with ACT.

Based on these results, drugs designation and development, such as modification and refinement, could be prospectively carried out to make combination of ligand and receptor more stable[30]. Since 297 these compounds were virtual screening and their inhibitory activities have not been reported, we will 298 conduct experiments such as IC50 and EC50 measurements in further studies to detect their biological 299 activities[21].

300 In this study, 4 modules of discovery studio 2016, including CBP pharmacophore, LibDock, 301 CDOCKER and molecular dynamics simulation, were used to screen and analyze the biochemical 302 structure characteristics of novel potential compounds. Molecular conformation, binding affinity and 
stability of the selected compounds were calculated and analyzed to determine their advantages over the

304 control compound act. A series of high-tech computational studies indicate that these 2 compounds may

305 have potential effects in inhibitors of ClCDA. Furthermore, our study provides guidance for the screening

306 of lead compounds with potential inhibitory effects. Through this method, more leading compounds

307 could be screened out, so as to improve the current inhibitor development and improve the efficiency of

308 inhibitor development.

309 Ethics approval and consent to participate: Not applicable.

310 Consent for publication: Not applicable.

311 Availability of data and material: We do not have any additional supporting data needed for this

312 manuscript. We included supporting data as a part of this manuscript as a separate file.

313 Competing interests: The authors declare no conflict of interest.

314 Funding: This work was supported by Applied Basic Research Program of Sichuan Province Sichuan

315 (No. 2019YJ0549), Sichuan Science and Technology Department Seedling engineering program (No.

316 201958), Sichuan Science and Technology program (No. 2019YFG0154).

317 Author Contributions: Conceptualization, Xiaoli Fu and Zizhong Tang; Data curation, Xiaoli Fu;

318 Formal analysis, Xiaoli Fu, Yujun Wan and Zizhong Tang; Investigation, Xiaoli Fu, Zizhong Tang and

319 Yirong Xiao; Methodology, Xiaoli Fu, Lu Huang, Haoxiang Wang and Biao Tang; Project administration,

320 Zizhong Tang; Resources, Hui Chen, Zizhong Tang, Huipeng Yao, Zhi Shan and Gang Wang;

321 Supervision, Zizhong Tang; Writing - original draft, Xiaoli Fu.

322 Acknowledgements: Not applicable.

\section{References}

324 1. Liu T, Liu Z, Song C, Hu Y, Zhifu H, She J, Fan F, Wang J, Jin C, Chang J, et al: Chitin-Induced

325 Dimerization Activates a Plant Immune Receptor. Science (New York, NY) 2012, 336:1160-1164.

326 2. Geoghegan I, Steinberg G, Gurr S: The Role of the Fungal Cell Wall in the Infection of Plants.

327 Trends in microbiology 2017, 25:957-967. 
328 3. Benhamou N, Broglie K, Broglie R, Chet I: Antifungal effect of bean endochitinase on Rhizoctonia solani: ultrastructural changes and cytochemical aspects of chitin breakdown. Can J Microbiol 1993, 39:318-328.

331 4. Deising H, Siegrist J: Chitin deacetylase activity of the rust Uromyces viciae-fabae is controlled by fungal morphogenesis. FEMS Microbiology Letters 1995, 127:207-211.

333 5. Gao F, Zhang B-S, Zhao J-H, Huang J-F, Jia P-S, Wang S, Zhang J, Zhou J-M, Guo H-S: Deacetylation of chitin oligomers increases virulence in soil-borne fungal pathogens. Nature plants 2019, 5:1167-1176.

6. El Gueddari NE, Rauchhaus U, Moerschbacher BM, Deising HB: Developmentally regulated conversion of surface-exposed chitin to chitosan in cell walls of plant pathogenic fungi. New Phytologist 2002, 156:103-112.

341 8. Masubuchi K, Taniguchi M, Umeda I, Hattori K, Suda H, Kohchi Y, Isshiki Y, Sakai T, Kohchi M, Shirai M, et al: Synthesis and structure-activity relationships of novel fungal chitin synthase

344 9. Lima SL, Colombo AL, de Almeida Junior JN: Fungal Cell Wall: Emerging Antifungals and Drug Resistance. Frontiers in Microbiology 2019, 10.

346 10. Zhao Y, Park R-D, Muzzarelli RA: Chitin deacetylases: properties and applications. Marine Drugs 2010, 8:24-46.

348 11. Freeman S, Katan T, Shabi E: Characterization of Colletotrichum species responsible for anthracnose diseases of various fruits. Plant disease 1998, 82:596-605. 
350 12. Dean R, Van Kan JA, Pretorius ZA, Hammond-Kosack KE, Di Pietro A, Spanu PD, Rudd JJ, 351 Dickman M, Kahmann R, Ellis J: The Top 10 fungal pathogens in molecular plant pathology. Molecular plant pathology 2012, 13:414-430.

353 13. Jaworska MM: Chitin deacetylase product inhibition. Biotechnology journal 2011, 6:244-247.

354 14. Jaworska M, Konieczna-Mordas E: Inhibition of chitin deacetylase by acetic acid, preliminary investigation. Progress on Chemistry and Application of Chitin and its Derivatives 2009, 14:83-88. enzymatic synthesis of $\boldsymbol{\beta}-\mathrm{D}-\mathrm{GlcNAc}-(\mathbf{1} \rightarrow \mathbf{4})-\mathrm{GlcN}$ from chitobiose. Carbohydrate research 1999 ,

16. Fu Y, Sun Y-N, Yi K-H, Li M-Q, Cao H-F, Li J-Z, Ye F: 3D pharmacophore-based virtual screening and docking approaches toward the discovery of novel HPPD inhibitors. Molecules

17. Blair DE, Hekmat O, Schüttelkopf AW, Shrestha B, Tokuyasu K, Withers SG, Van Aalten DM: Structure and mechanism of chitin deacetylase from the fungal pathogen Colletotrichum lindemuthianum. Biochemistry 2006, 45:9416-9426.

18. Li P, Peng J, Zhou Y, Li Y, Liu X, Wang L, Zuo Z: Discovery of FIXa inhibitors by combination of pharmacophore modeling, molecular docking, and 3D-QSAR modeling. J Recept Signal Transduct Res 2018, 38:213-224. Virtual Screening, Docking and Density Functional Theory Approach Towards the Discovery 
20. Zhou X, Yu S, Su J, Sun L: Computational study on new natural compound inhibitors of pyruvate dehydrogenase kinases. International journal of molecular sciences 2016, 17:340.

21. Hu W, Kumar J, Tsai JJ: Computational Analysis of Potential Inhibitors Selected Based On Structural Similarity for the Src SH2 Domain. International Journal of Mechanical and Mechatronics Engineering 2014, 8:365-368.

22. Zhang Y, Zhang S, Xu G, Yan H, Pu Y, Zuo Z: The discovery of new acetylcholinesterase inhibitors derived from pharmacophore modeling, virtual screening, docking simulation and bioassays. Molecular BioSystems 2016, 12:3734-3742.

23. Alam S, Khan F: Virtual screening, Docking, ADMET and System Pharmacology studies on Garcinia caged Xanthone derivatives for Anticancer activity. Scientific Reports 2018, 8:5524.

24. Rajesh D, Muthukumar S, Saibaba G, Siva D, Akbarsha MA, Gulyás B, Padmanabhan P, Archunan G: Structural elucidation of estrus urinary lipocalin protein (EULP) and evaluating binding affinity with pheromones using molecular docking and fluorescence study. Scientific Reports 2016, 6:35900.

25. Yamada M, Kurano M, Inatomi S, Taguchi G, Okazaki M, Shimosaka M: Isolation and characterization of a gene coding for chitin deacetylase specifically expressed during fruiting body development in the basidiomycete Flammulina velutipes and its expression in the yeast Pichia pastoris. FEMS Microbiology Letters 2008, 289:130-137.

26. Peng J, Li Y, Zhou Y, Zhang L, Liu X, Zuo Z: Pharmacophore modeling, molecular docking and molecular dynamics studies on natural products database to discover novel skeleton as nonpurine xanthine oxidase inhibitors. J Recept Signal Transduct Res 2018, 38:246-255. 
393 27. Verdonk ML, Cole JC, Hartshorn MJ, Murray CW, Taylor RD: Improved protein-ligand docking using GOLD. Proteins 2003, 52:609-623. Mycobacterium tuberculosis MurG: Homology Modelling, Structure Based Pharmacophore, Molecular Docking, and Molecular Dynamics Simulations. Journal of Biomolecular Structure E Dynamics 2017, 36:1-42.

399 29. Sakkiah S, Kusko R, Pan B, Guo W, Ge W, Tong W, Hong H: Structural Changes Due to Antagonist Binding in Ligand Binding Pocket of Androgen Receptor Elucidated Through Molecular Dynamics Simulations. Frontiers in Pharmacology 2018, 9. 


\section{Figures}

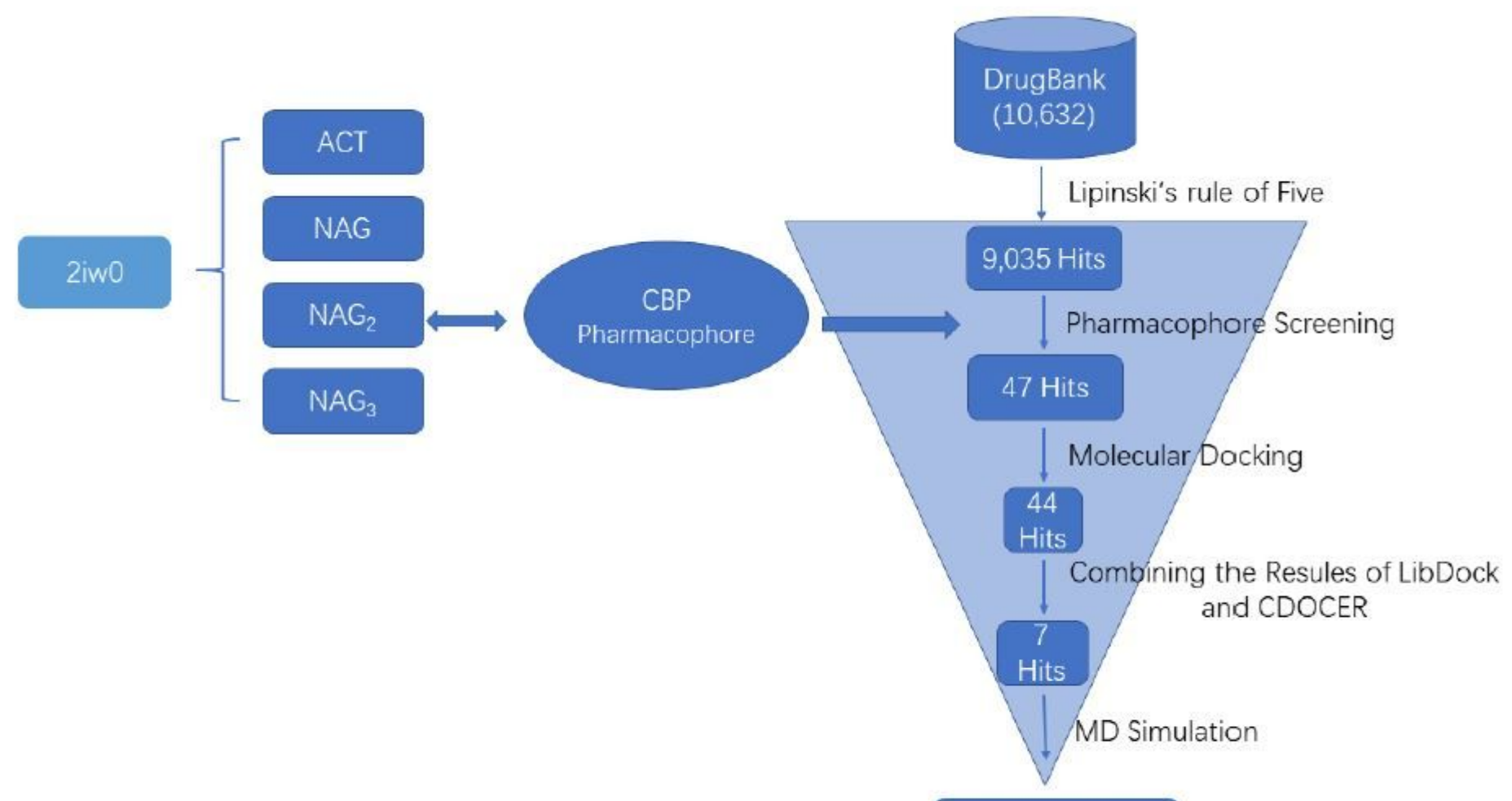

2 Potential Hits

\section{Figure 1}

Schematic representation of the virtual screening process implemented in the identification of CICDA inhibitors. 2iw0: crystal structure of CICDA; ACT: acetate ion; NAG: chitin monomer form; NAG2: chitin dimer form; NAG3: trimeric form of chitin. 


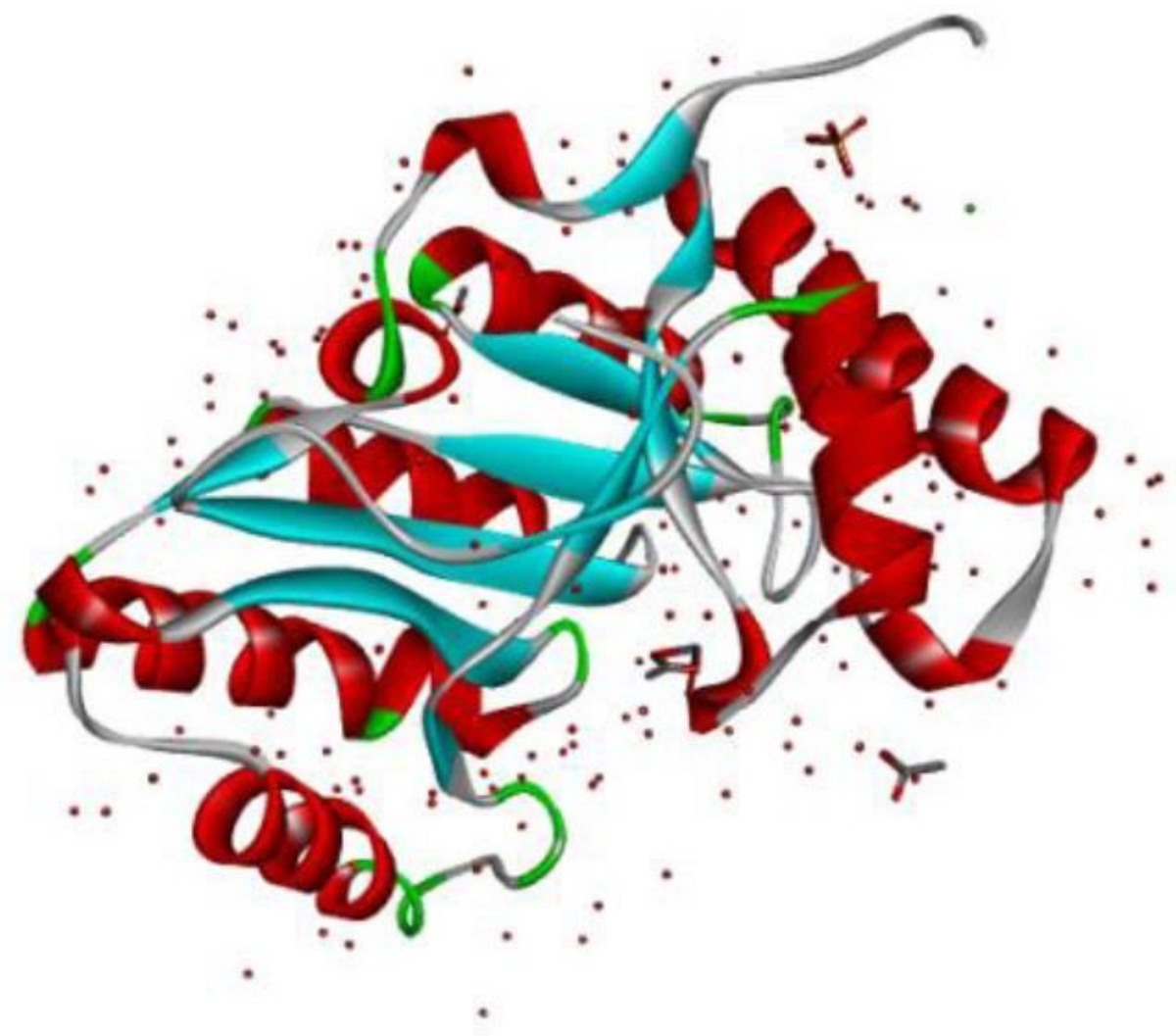

Figure 2

Crystal structure of chitin deacetylase (2iw0). 


\section{H-Bonds}
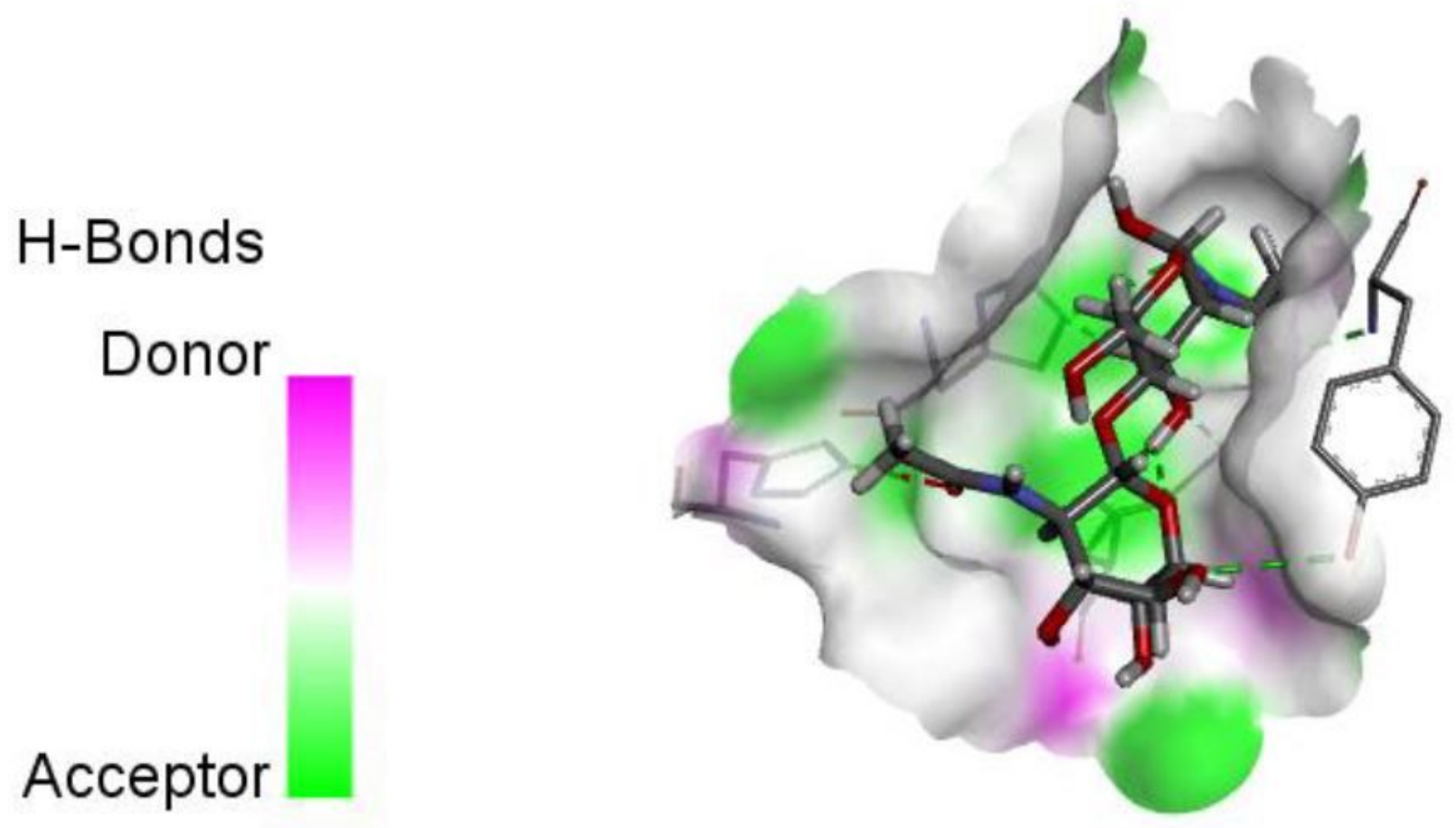

Figure 3

Hydrogen bond diagram of 2iw0-NAG2 interaction.

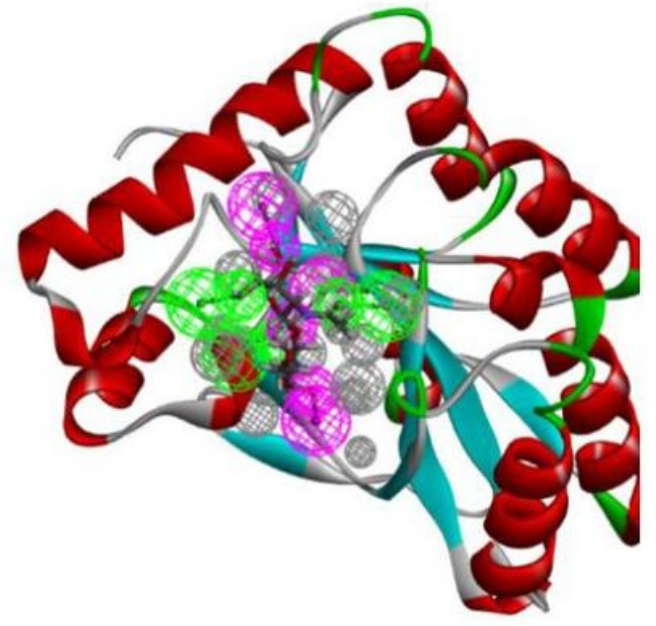

(A)

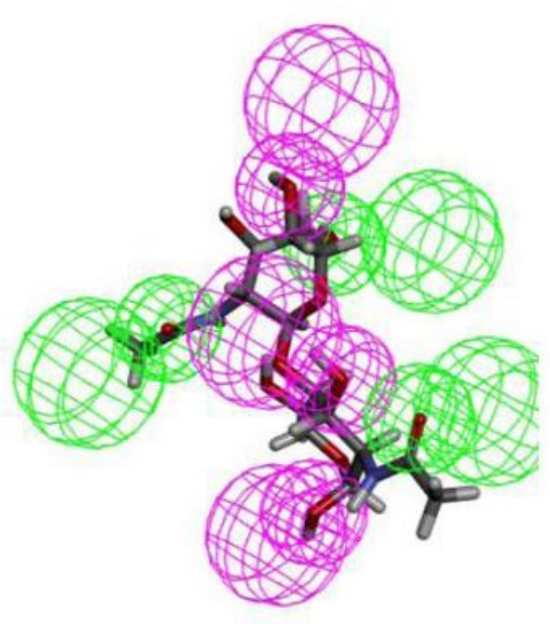

(B)

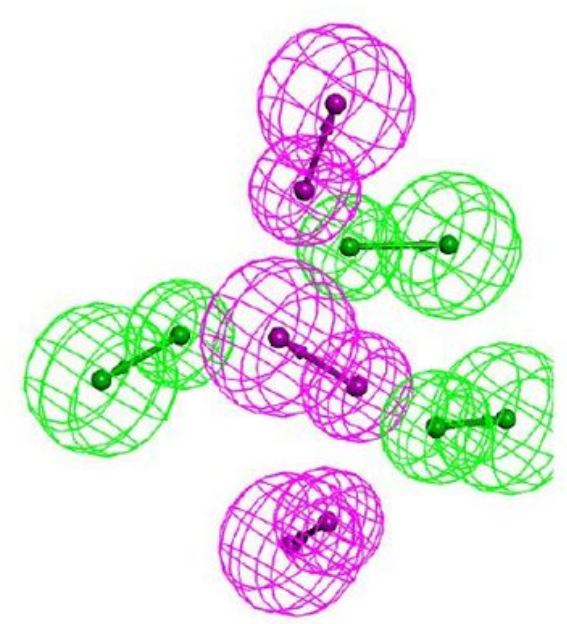

(C)

Figure 4

(A) Chitin deacetylase crystals (PDB code: 2iw0) with interaction diagram of co-crystalline ligand NAG2 and pharmacophore_01, (B) co-crystalline ligand NAG2 and pharmacophore_01, (C) pharmacophore_01. 


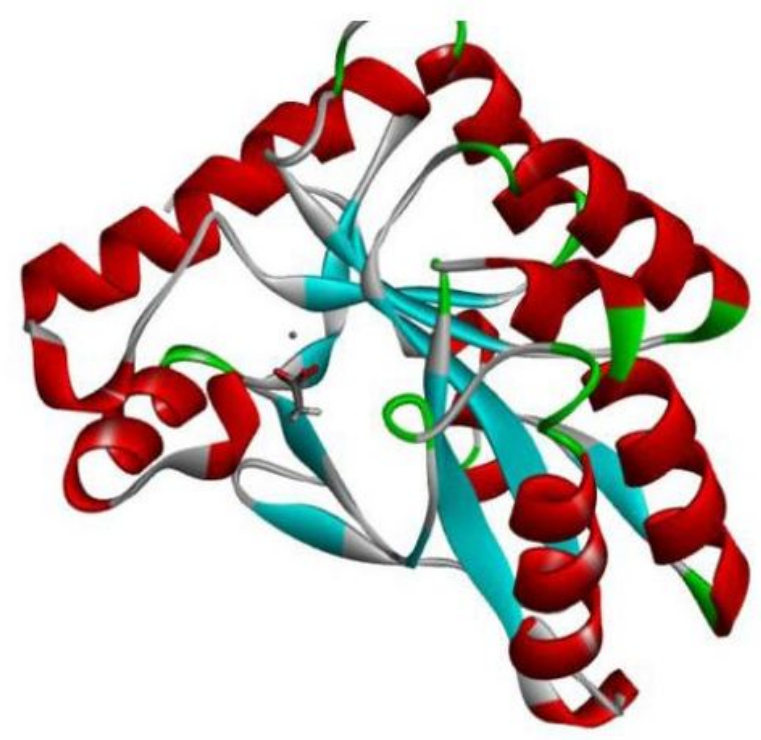

(A)

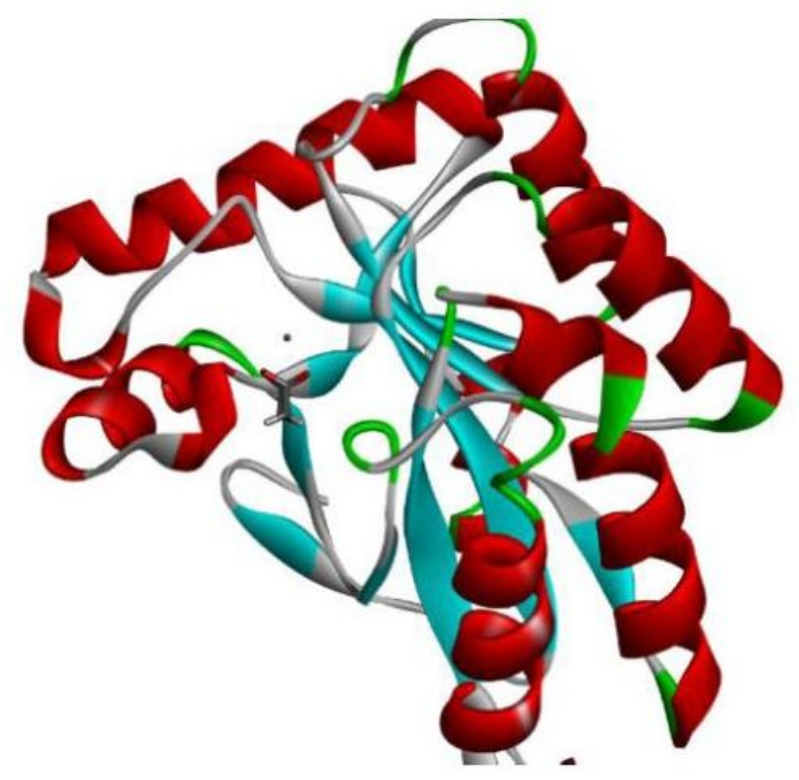

(B)

\section{Figure 5}

Alignment of the docked ligands with the ligands in the crystallographic complex. (A) The ligand by the LibDock docking method; (B) The ligand by the CDOCKER docking method. 


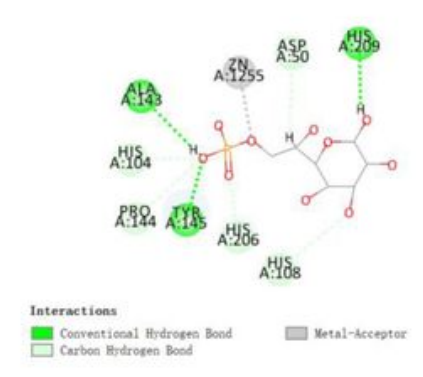

(A)

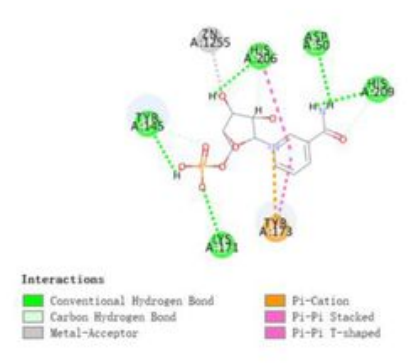

(C)

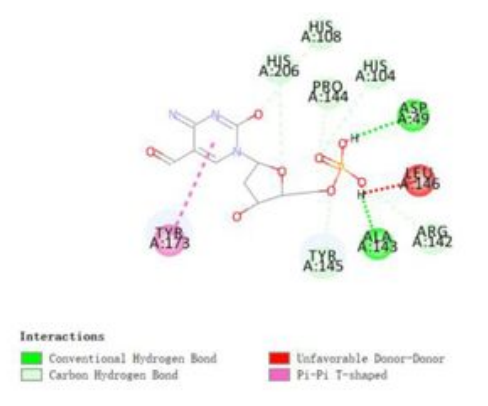

(E)
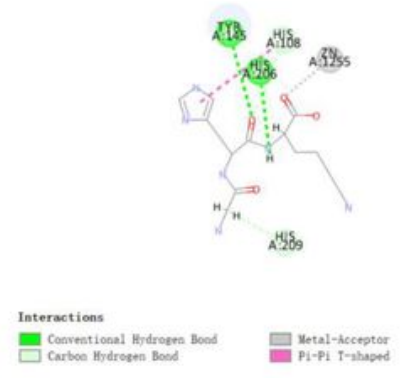

(G)

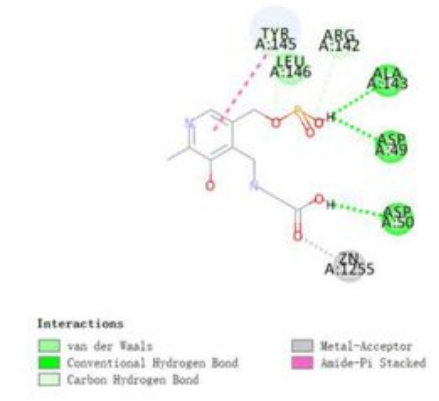

(B)

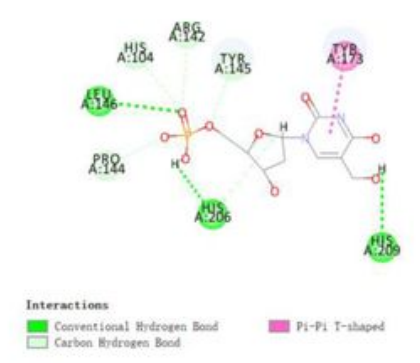

(D)

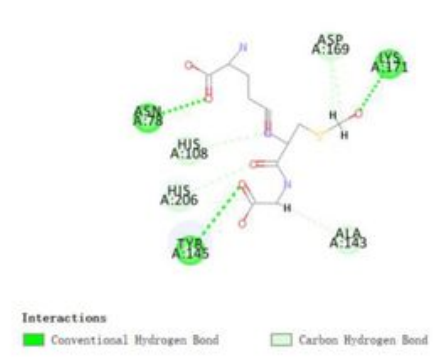

(F)

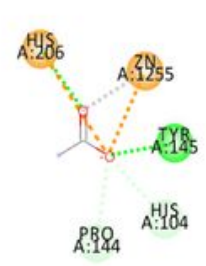

Interactions
Attractive Charge
Conventional Hydrogen Bond $\square$ Carbon Hydrogen Bond
Netal-Acceptor

(H)

\section{Figure 6}

The receptor-ligand interaction of screening compound with the 2iw0 active site. (A) DB02470 Receptorligand interaction with 2iw0 active site. (B) DB02824 Receptor-ligand interaction with 2iw0 active site. (C) DB03227 Receptor-ligand interaction with 2iw0 active site. (D) DB03846 Receptor-ligand interaction with 2iw0 active site. (E) DB04603 Receptor-ligand interaction with 2iw0 active site. (F) DB05446 Receptor- 
ligand interaction with 2iw0 active site. (G) DB11296 Receptor-ligand interaction with 2iw0 active site. (H) ACT Receptor-ligand interaction with 2iw0 active site.

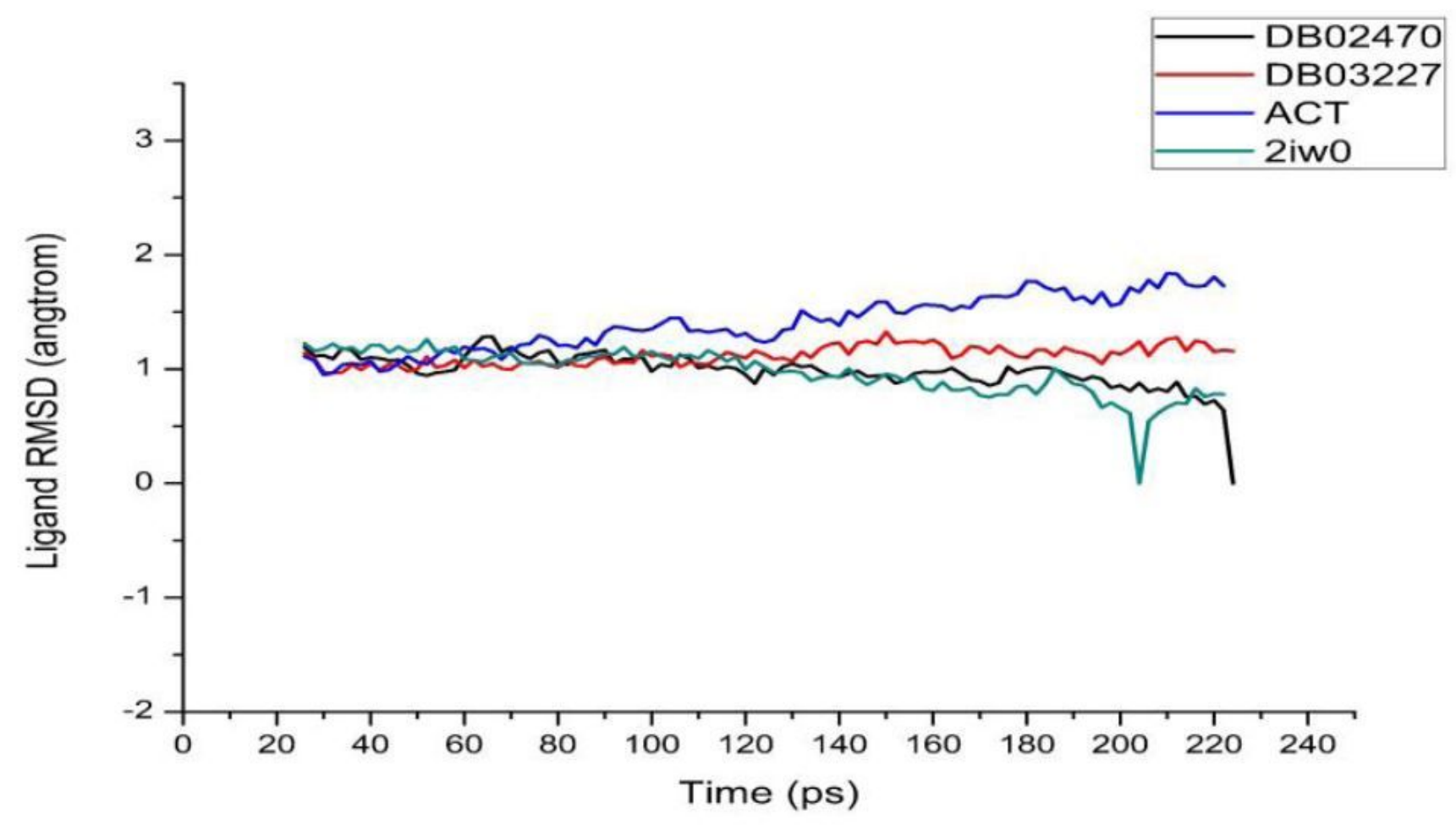

Figure 7

The RMSD values of protein-ligand complexes during MD simulation. 


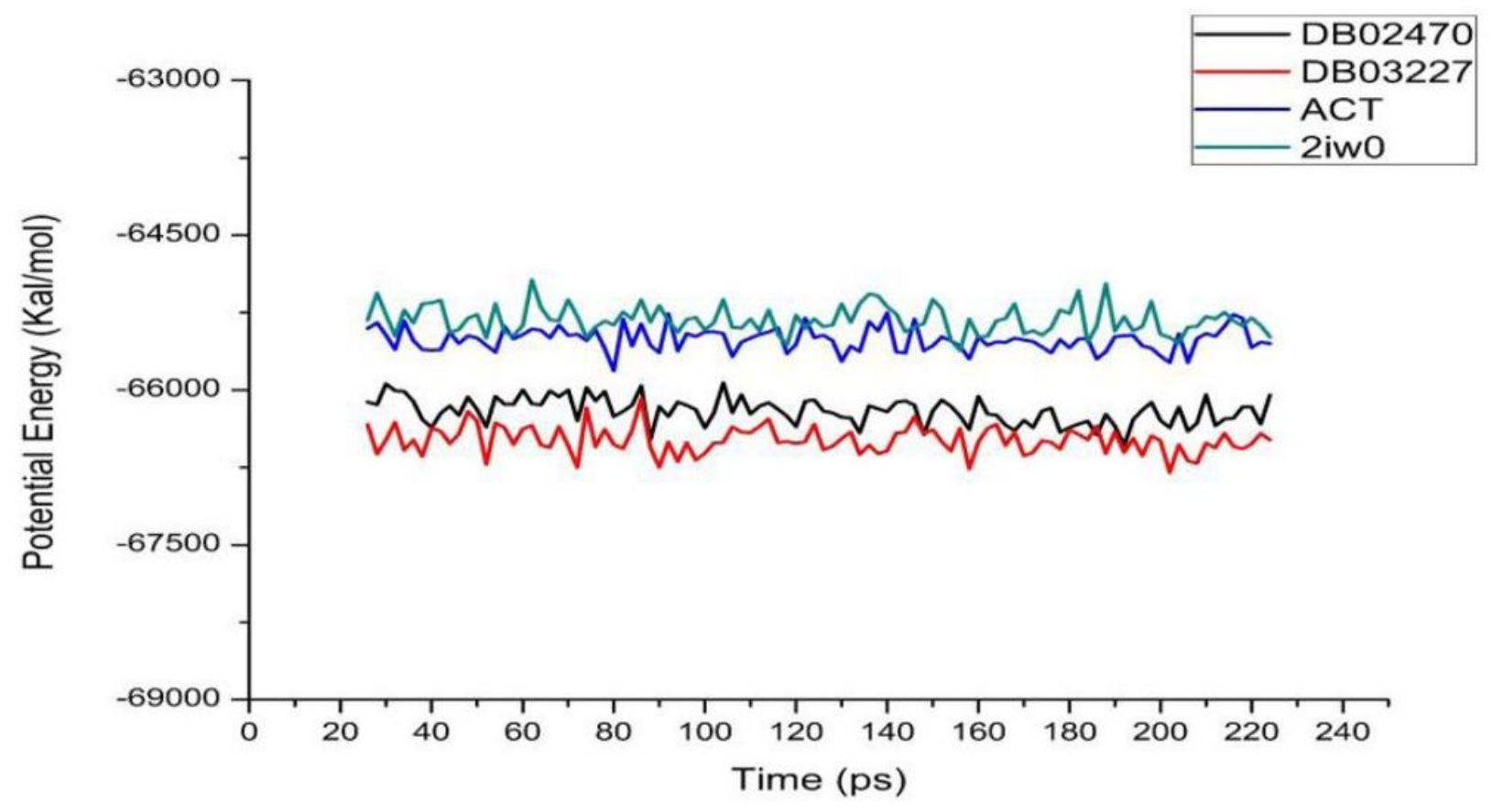

Figure 8

Potential energies of protein-ligand complexes during MD simulation.

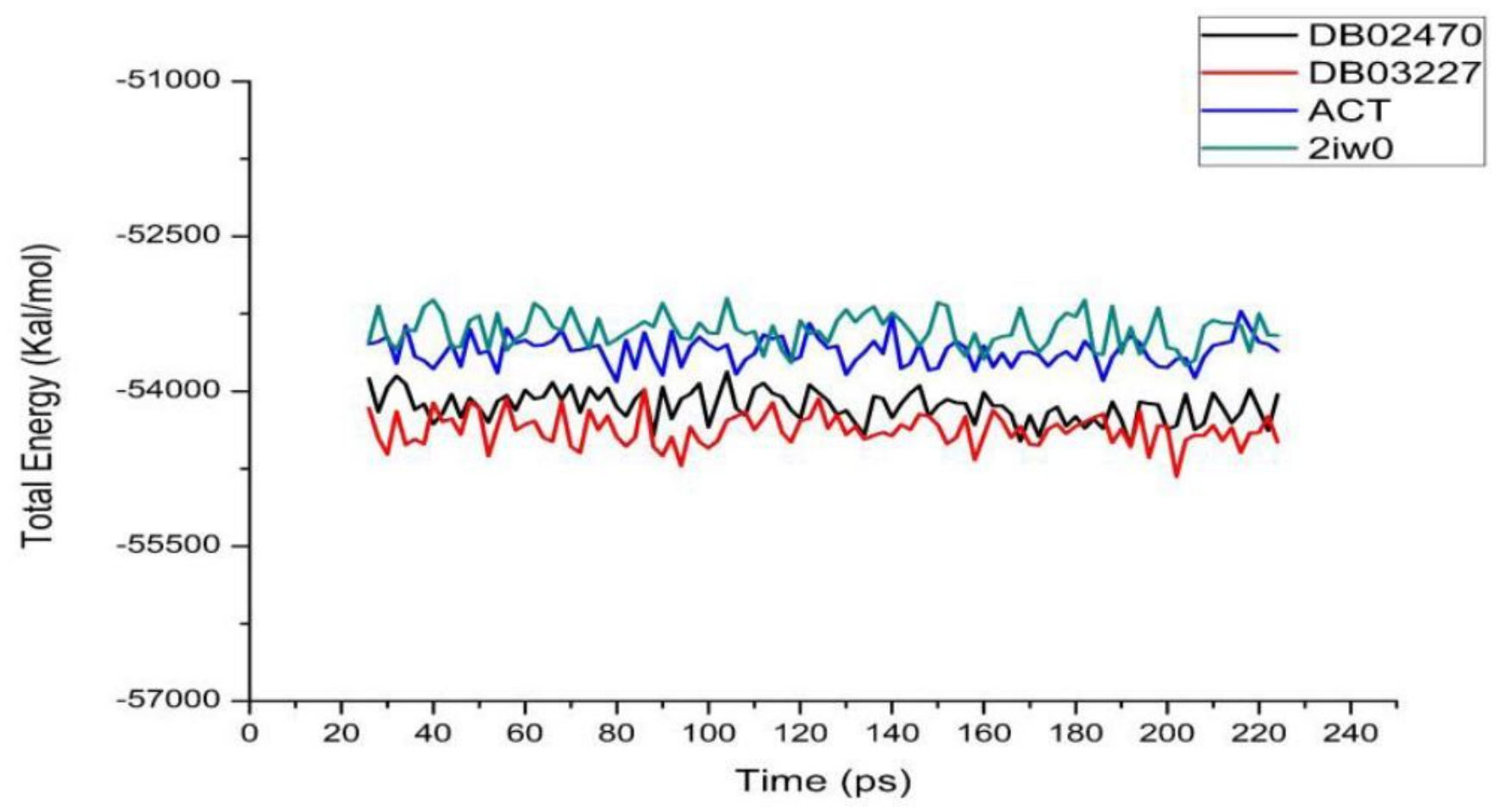

Figure 9 
Total energies of protein-ligand complexes during MD simulation.
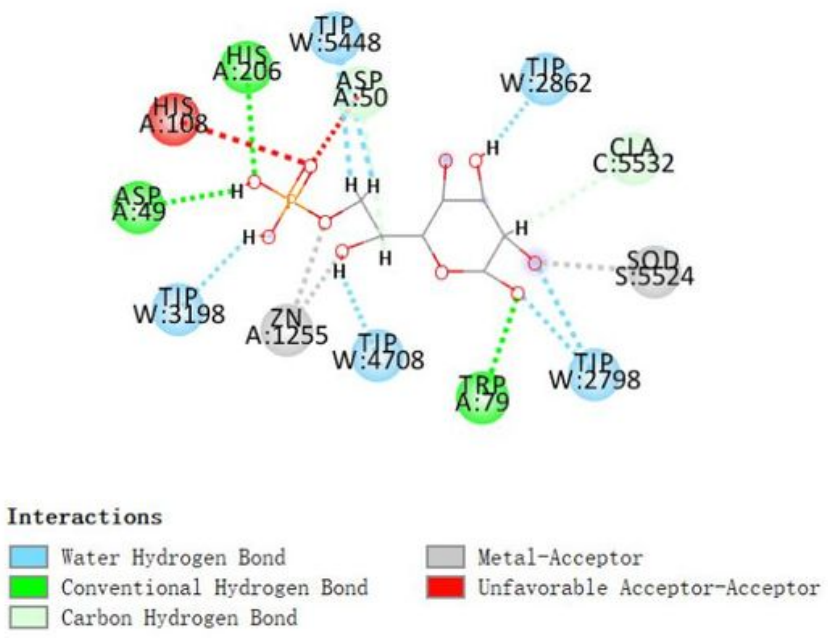

(A)

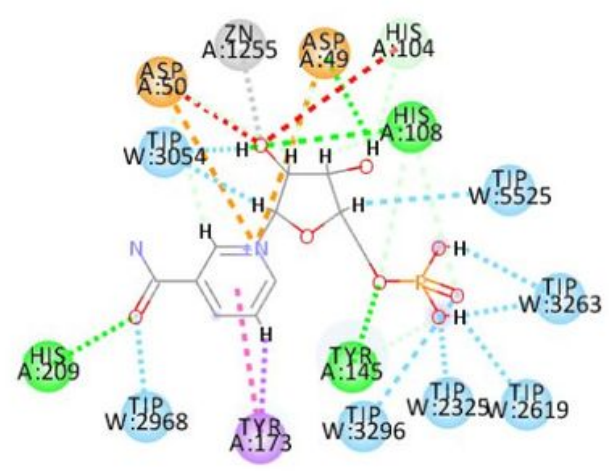

\section{Figure 10}

The compounds were screened for receptor-ligand interaction with the 2iw0 active site after MD simulation. DB02470 on the left and DB03227 on the right. 